\title{
Clinical and comparative utility of afatinib in non-small cell lung cancer
}

\author{
This article was published in the following Dove Press journal: \\ Biologics: Targets and Therapy \\ 23 April 2014 \\ Number of times this article has been viewed
}

\author{
Manolo D'Arcangelo \\ Fred R Hirsch \\ University of Colorado Denver, \\ Department of Medical Oncology, \\ Aurora, CO, USA
}

\begin{abstract}
The first targeted agents approved for non-small cell lung cancer (NSCLC) treatment, the epidermal growth factor receptor (EGFR) tyrosine kinase inhibitors (TKIs) gefitinib and erlotinib, have an impressive activity in the presence of activating mutations of the EGFR gene. However, all patients develop acquired resistance principally through secondary mutations (T790M), HER2 amplification, MET amplification, and other molecular aberrations. An attempt to overcome EGFR TKI resistance has been through the development of irreversible blockers. Afatinib is an irreversible inhibitor of the tyrosine kinase activity of all members of the HER family. The pharmacologic properties of afatinib (formation of covalent bonds, inhibition of other family members, and in vitro and in vivo activity on T790M mutation positive tumors) made this drug particularly appealing to study in clinic. Therefore, an intense program of clinical research (LUX-Lung program) was started and clinical results have shown very encouraging activity profiles in patients harboring EGFR activating mutations and in those with acquired resistance to reversible TKIs.
\end{abstract}

Keywords: NSCLC, EGFR, tyrosine kinase inhibitor, afatinib

\section{Introduction}

The inhibition of the epidermal growth factor receptor (EGFR), a tyrosine kinase receptor commonly overexpressed or aberrantly active in epithelial cancers, has been shown to be a successful strategy in the treatment of patients with non-small cell lung cancer (NSCLC). ${ }^{1,2}$ Based on the results of several Phase III clinical trials, ${ }^{3-8}$ erlotinib and gefitinib were the first two targeted agents to be approved for clinical use in NSCLC all over the world with diverse indications. These drugs are two small molecules that selectively inhibit the tyrosine kinase activity of the receptor (tyrosine kinase inhibitors, TKIs). Erlotinib and gefitinib belong to the first generation of TKIs and have reversible binding features. The principal predictive factor for response to such drugs is the presence of somatic "activating" mutations in the EGFR gene that cause an aberrant and constitutive activation of the receptor at the membrane level, resulting in a growth advantage of tumor cells through the activation of Ras/MAPK, PI3K/Akt, and STAT signaling pathways. ${ }^{2}$ In the specific group of NSCLC patients bearing EGFR mutation (about 10\%-15\% of Caucasian and up to $40 \%$ of Asian patients) treatment with EGFR TKIs provides higher response rates (55\% up to 85\%), longer progressionfree survival (PFS, from 8.4 months to 13.1 months), and better quality of life (QoL) compared to classical chemotherapy. ${ }^{3-8}$ Although about $70 \%$ of patients are likely to respond to the anti-EGFR treatment, virtually all of them progress after a median of 9-12 months from treatment commencement. ${ }^{3-8}$ Rebiopsy based research programs
Correspondence: Fred R Hirsch University of Colorado Cancer Center, I280I E 17th Avenue, Mail Stop 8II7, Aurora, CO, USA

Tel +I 3037243858

Email fred.hirsch@ucdenver.edu 
have identified several mechanisms through which EGFRaddicted lung tumors can overcome the beneficial effect of TKIs. In about $50 \%$ of cases, a secondary mutation (T790M) is supposed to be responsible for resistance. ${ }^{9,10}$ Other mechanisms involve MET gene amplification (5\%-20\%), ${ }^{11} \mathrm{PI} 3 \mathrm{KCA}$ gene mutations (5\%), HER2 gene amplification (13\%), ${ }^{12}$ and small-cell histologic transformation $(5 \%-15 \%) .{ }^{12-13}$ Overlap among mechanisms of acquired resistance may be observed in a small percentage of tumors. ${ }^{12}$ However, in $30 \%$ of cases, a clear mechanism of acquired resistance remains unknown.

Several approaches to overcome acquired resistance are under investigation. The first and more intuitive attempt has been combining reversible EGFR TKIs to other target agents, such as anti-MET antibodies and small molecules, mTOR blockers, anti-HER2 drugs, and histone deacetylase (HDAC) inhibitors. Dual pathway inhibition through multi-target drugs is also being explored. Another attempt to overcome resistance is through irreversible EGFR TKIs, which are small molecules forming covalent bonds to EGFR and have the characteristic of inhibiting HER2 as well. The rationale for the development of this type of inhibitors is strong. The mutation T790M is localized at codon 790 on exon 20 of EGFR and results in the change of threonine to methionine at the protein level. The amino acid change causes steric hindrance to EGFR TKIs in crystal analysis and/or increased affinity for adenosine triphosphate (ATP). ${ }^{14,15}$ Consequently, reversible EGFR TKIs can no longer bind to the receptor or cannot efficiently compete for the ATP-binding site. Recent studies suggest that the mutation may be present within tumors before TKI treatment and that the clones harboring such a mutation may become dominant after TKI selection pressure. ${ }^{16,17}$ On the basis of these observations, irreversible EGFR TKIs were developed with the aim of overcoming the molecular aberration through the formation of covalent bonds in the pocket of the catalytic site.

Afatinib (BIBW 2992; Boehringer-Ingelheim Pharma, Ingelheim, Germany) is an irreversible pan-HER inhibitor that blocks all the members of the HER family with tyrosine kinase properties (EGFR, HER2, and HER4). In vitro and in vivo preclinical studies showed interesting antitumor activity of this compound. Consequently, an intense program of clinical research (LUX-Lung program) was developed (Table 1) and is currently ongoing in several categories of NSCLC patients (EGFR-mutated and wild type tumors; reversible EGFR TKIs naïve or resistant patients; adenocarcinoma and squamous cell carcinoma histology). The first four clinical trials were published and ongoing reports are available for others. In June 2013, based on the results of LUX-Lung 3, the US Food and Drug Administration (FDA) approved afatinib for first-line treatment of patients with EGFR-mutated NSCLC.

The aim of the present article is to critically review the clinical trials exploring afatinib efficacy in lung cancer and to compare, when possible, this novel agent to available treatments.

\section{Afatinib pharmacology Pharmacodynamics}

Afatinib is an ATP-competitive aniline-quinazoline compound with a reactive acrylamide group that irreversibly binds to cysteine residues within the kinase domain of EGFR and HER2 (Cys797 and Cys805, respectively). ${ }^{18}$ The structure of afatinib is shown in Figure 1. A more recent study showed that afatinib is also an irreversible inhibitor of HER4, binding the cysteine in position $803 .{ }^{19}$ The half maximal effective concentration $\left(\mathrm{EC}_{50}\right)$ values for EGFR, HER2, and HER4 are $0.5,14$ and $1 \mathrm{nM}$, respectively. ${ }^{19}$ Compared to erlotinib and gefitinib, afatinib shows lower $\mathrm{EC}_{50}$ values and greater

Table I LUX-Lung clinical development program

\begin{tabular}{|c|c|c|c|c|c|}
\hline $\begin{array}{l}\text { LUX-Lung } \\
\text { study }\end{array}$ & Phase & $\begin{array}{l}\text { Enrollment } \\
\text { (patients, n) }\end{array}$ & Treatment & Target population & $\begin{array}{l}\text { Primary } \\
\text { end point }\end{array}$ \\
\hline I & $\mathrm{Ilb} / \mathrm{III}$ & 585 & Afatinib vs placebo & I-2 CT lines and erlotinib/gefitinib-resistant & OS \\
\hline 2 & II & 129 & Afatinib & EGFR mut+, 0-I prior CT line & $\mathrm{RR}$ \\
\hline 3 & III & 330 & Afatinib vs CDDP + PEM & EGFR mut+, treatment naïve & PFS \\
\hline 4 & $\mathrm{I} / \mathrm{II}$ & 72 & Afatinib & I-2 CT lines and erlotinib/gefitinib-resistant & $\begin{array}{l}\text { Phase I: safety } \\
\text { Phase II: RR }\end{array}$ \\
\hline 5 & III & $\mathrm{I}, 155$ & Afatinib + TXL vs CT & Erlotinib/gefitinib-resistant, afatinib-resistant & PFS \\
\hline 6 & III & 364 & Afatinib vs CDDP + Gem & EGFR mut+, treatment naïve & PFS \\
\hline 7 & Ilb & 316 & Afatinib vs gefitinib & EGFR mut + , treatment naïve & PFS, TTP, OS \\
\hline 8 & III & 800 & Afatinib vs erlotinib & I prior CT line, SqCC histology & PFS \\
\hline
\end{tabular}

Abbreviations: CDDP, cisplatin; CT, chemotherapy; EGFR mut+, epidermal growth factor mutation positive; Gem, gemcitabine; OS, overall survival; PEM, pemetrexed; PFS, progression-free survival; RR, response rate; SqCC, squamous cell carcinoma; TTP, time to progression; TXL, paclitaxel. 


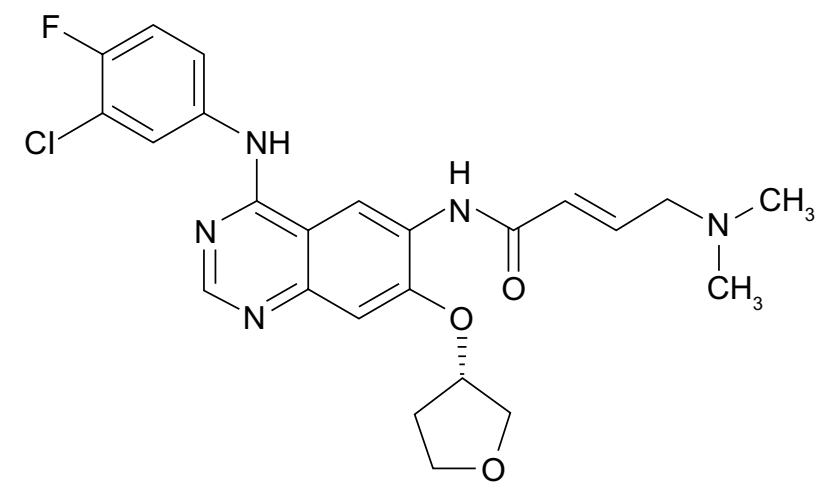

Figure I Afatinib chemical structure.

potency against wild type (afatinib vs erlotinib vs gefitinib: $60 \mathrm{nM}$ vs $110 \mathrm{nM}$ vs $157 \mathrm{nM}$ ) and L858R-mutated EGFR (afatinib vs erlotinib vs gefitinib: $0.7 \mathrm{nM}$ vs $40 \mathrm{nM}$ vs $5 \mathrm{nM}$ ). ${ }^{18}$ Moreover, afatinib is able to inhibit the kinase activity in vitro and in animal models when a resistant T790M mutation is concomitant to a sensitivity mutation. ${ }^{18} \mathrm{The}^{\mathrm{EC}}$ in this case is $99 \mathrm{nM}$.

The pharmacologic properties of afatinib make this compound particularly attractive for overcoming resistance to first-generation TKIs. First, afatinib binds ErbB family members with covalent bonds and this translates into a complete and sustained abolition of the kinase activity of the receptor. Moreover, afatinib is active in vitro and in vivo in the presence of "secondary" mutations, a mechanism responsible for $50 \%-60 \%$ of cases of resistance. ${ }^{9}{ }^{10}$ Finally, afatinib inhibits HER2, which is the preferential dimerization partner of EGFR and whose gene amplification is responsible for acquired EGFR TKI resistance in $10 \%-15 \%$ of patients. ${ }^{12}$ These findings supported the clinical development of this novel agent both in EGFR-mutated and wild type patients and in EGFR TKI naïve and pretreated patients.

\section{Pharmacokinetics}

The first study exploring afatinib pharmacokinetics was conducted on eight healthy male volunteers, who received a single oral dose of radiolabeled afatinib (corresponding to $22.2 \mathrm{mg}$ of the formulation used in clinic). ${ }^{20}$ Excretion of afatinib was principally via feces $(85.4 \%) .{ }^{20}$ Absorption was slow, with maximum plasma concentrations reached 6 hours after administration. The mean terminal half-life was 33.9 hours in plasma. ${ }^{20}$ Authors found a high volume of distribution, indicating a high capacity of the compound to penetrate into tissues. Metabolism of afatinib was minimal, and in particular, oxidative metabolism by cytochrome P-450 was negligible. ${ }^{20}$
Another Phase I study evaluated afatinib in 53 solid tumor patients who received the drug at $10-50 \mathrm{mg}$ daily. Afatinib was well-tolerated with dose-limiting toxicities reported in only three patients (two with skin rash and one with pneumonitis). ${ }^{21}$ The recommended Phase II dose was $50 \mathrm{mg}$ daily. In a subgroup of patients $(\mathrm{n}=13)$, the effect of food on pharmacokinetic parameters was evaluated. Patients received afatinib at $40 \mathrm{mg}$ daily after overnight fasting or a high-fat content breakfast. Food intake before dosing sensibly decreased afatinib disposition; therefore, the authors suggested that the drug is best administered after fasting. ${ }^{21}$

A recent meta-analysis including data from four Phase I trials and one Phase II trial showed moderate intraindividual variability in afatinib trough concentration values and moderate-to-high inter-patient variability in plasma concentrations..$^{22}$ The exposure to afatinib (as measured by the area under the curve $[\mathrm{AUC}]$ and peak concentration $\left[\mathrm{C}_{\max }\right]$ ) correlated with the severity of the most common adverse events (diarrhea and rash). ${ }^{22}$ This may explain the great variability in toxicity profile observed in clinic.

\section{Efficacy studies \\ Trials in first-line treatment of EGFR-mutated NSCLC}

One Phase II trial and three Phase III trials are investigating afatinib in EGFR-mutated NSCLC. ${ }^{23-25}$ LUX-Lung 2 was a single arm Phase II trial that assessed the activity of two doses of afatinib (40 mg and $50 \mathrm{mg}$ daily) as first- or second-line treatment of 129 NSCLC patients harboring EGFR mutations. ${ }^{23}$ Ninety-nine patients started afatinib at $50 \mathrm{mg}$ and 30 patients at $40 \mathrm{mg}$. A complete or partial response was observed in 79 patients $(61 \%)$, with no significant difference according to the starting dose. ${ }^{23}$ As no apparent difference in activity was highlighted between the two groups of patients, $40 \mathrm{mg}$ dosing was chosen for Phase III trials in early line setting. No significant difference was observed between patients receiving the experimental drug as first- or second-line treatment (66\% vs 57\%, OR: 0.71, 95\% confidence interval [CI]: 0.35-1.44) ${ }^{23}$ Median PFS was 10.1 months and overall survival (OS) was 24.8 months for all patients. ${ }^{23}$ Notably, OS was 23.3 months for patients receiving afatinib as second-line treatment, while the number of events to calculate median OS is still insufficient in the first-line group, suggesting a potentially significant difference in survival if afatinib is given as first or successive lines of treatment of $E G F R$-mutation-positive patients.

The LUX-Lung 3 and LUX-Lung 6 Phase III trials confirmed the interesting activity of afatinib and assessed its superiority compared to standard chemotherapy as front-line 
therapy in the presence of EGFR mutations. ${ }^{24,25}$ In LUXLung 3, 345 patients were randomized (2:1 ratio) to receive afatinib $40 \mathrm{mg}$ daily $(\mathrm{n}=230)$ or a combination of cisplatin and pemetrexed every 21 days $(n=115) .{ }^{24}$ Patients were stratified by mutation type (exon 19 deletion, L858R, or other) and race (Asian or non-Asian). Median PFS was 11.1 months for afatinib compared to 6.9 months for chemotherapy (HR: 0.58; 95\% CI: $0.43-0.78 ; P=0.001) .{ }^{24}$ A slightly better PFS of 13.6 months was detected in patients $(n=308)$ with common activating mutations (exon 19 deletions and exon 21 point mutations) compared to the whole population. Patients also benefitted more from afatinib than chemotherapy in terms of response rate (56\% and $23 \%$, respectively). ${ }^{24}$ It is widely recognized that with target therapies, standardization of molecular testing assay and methodology is important to define the population to treat and assure reproducibility of results. Therefore, in LUX-Lung 3, EGFR status assessment was performed in central laboratories using a standardized allele-specific quantitative real-time polymerase chain reaction kit (Therascreen EGFR 29; Qiagen, Manchester, UK) that can detect up to 29 somatic EGFR mutations in paraffinembedded tumors. Given the positive results of LUX-Lung 3, the therascreen $E G F R$ test received approval to market in the US, and currently, the FDA afatinib label requires this diagnostic assay. ${ }^{26}$

Efficacy of afatinib in EGFR-mutation-positive patients was confirmed in the LUX-Lung 6 trial in 364 Asian patients. ${ }^{25}$ This study had the same design of LUX-Lung 3, differing only in the control chemotherapy regimen (cisplatin and gemcitabine) chosen for the unavailability of pemetrexed in some Asian countries. Recruitment is currently closed and preliminary data were presented at a poster session during the ASCO 2013 annual meeting. ${ }^{25}$ Patients receiving afatinib compared to chemotherapy had a significantly prolonged PFS (11 vs 5.6 months, HR: 0.28, 95\% CI: 0.20-0.39; $P<0.0001)$ as well as higher response rate (RR; 66.9 vs $23 \% ; P<0.0001) .^{25}$

Recently, the analysis of afatinib efficacy in patients with uncommon EGFR mutations enrolled in LUX-Lung 2, 3, and 6 was presented at the World Conference of Lung Cancer $2013 .{ }^{27}$ Of the 838 patients enrolled, 100 were found to harbor an uncommon EGFR mutation (de novo T790M, exon 20 insertions, and other) and 75 were treated with afatinib (Table 2). The enrollment of patients with uncommon mutations may have jeopardized the benefit reported for afatinib. Indeed, when patients with uncommon mutations are excluded, the PFS is prolonged from 11 to 13.6 months. However, given the interesting pharmacologic properties of
Table 2 Afatinib in patients with uncommon EGFR mutations in the LUX-Lung 2, 3, and 6 trials $^{27}$

\begin{tabular}{lllll}
\hline EGFR mutation & $\begin{array}{l}\text { Patients } \\
(\mathbf{n})\end{array}$ & RR (\%) & $\begin{array}{l}\text { PFS } \\
\text { (months) }\end{array}$ & $\begin{array}{l}\text { OS } \\
\text { (months) }\end{array}$ \\
\hline Exon 19 del + & 308 & 60.8 & 13.6 & $\mathrm{NR}$ \\
Exon 2I point mut & & & & \\
Exon 20 insertion & 20 & 8.7 & 2.7 & 9.4 \\
De novo T790M & 14 & 14.3 & 2.9 & 14.9 \\
G7I9X (exon 18) & 18 & 78.0 & 13.8 & 26.9 \\
L86IQ (exon 20) & 16 & 56.0 & 8.2 & 16.9 \\
S768I (exon 21) & 5 & 100 & 14.7 & $\mathrm{NR}$ \\
\hline
\end{tabular}

Abbreviations: del, deletion; EGFR, epidermal growth factor; mut, mutation; n, number; NR, not reported; PFS, progression free survival; OS, overall survival; $R R$, response rate.

afatinib, the enrollment of such patients was necessary to assess afatinib efficacy in this subgroup of NSCLC. One may argue that ad hoc studies in this subpopulation of patients should have been planned. However, in the group harboring exon 20 insertion or de novo T790M mutation, RR, PFS, and OS were extremely poor. ${ }^{27}$ For other uncommon mutations, including G719X and L861Q, which show limited sensitivity to reversible EGFR TKIs, ${ }^{28}$ RR and PFS were notably improved. ${ }^{27}$ These findings open the possibility for patients with G719X and L861Q mutations to be treated with a targeted agent, although further studies are warranted to develop a true, strong recommendation. Patients with other uncommon mutations should be best enrolled in trials with novel agents or treated with chemotherapy.

The LUX-Lung 3 and 6 trials join a series of other trials comparing an EGFR TKI to chemotherapy for the front-line treatment of NSCLC with EGFR mutations. All these trials demonstrated a statistically significant and clinically meaningful difference in RR and PFS, although no study has demonstrated an OS improvement, which is likely due to extensive crossover between treatment arms. $^{3-8}$ However, LUX-Lung 3 and 6 are the two largest registration trials conducted to date in EGFR-mutated patients, which strengthens the significance of their results. Moreover, LUX-Lung 3 is the only trial that used the real standard first-line treatment (cisplatin and pemetrexed) for the control arm rather than a platinum combination with taxanes or gemcitabine. Indeed, the PFS in the control arm is the highest ever registered in trials comparing EGFR TKIs to chemotherapy.

Given the approval of three targeted agents for $E G F R$ mutation-positive NSCLC (erlotinib, gefitinib, and afatinib) with regulatory differences from country to country, a question is inescapable: is afatinib better than reversible TKIs for EGFR-mutated NSCLC patients? Although 
a clinical trial comparing afatinib to gefitinib is already ongoing (LUX-Lung 7), ${ }^{29}$ no data are currently available. Comparisons between trials may be only speculative and may result in false conviction due to differences in trial designs, inclusion/exclusion criteria, methods, and statistical analysis, etc. With these premises, afatinib seems to perform better than erlotinib and gefitinib in terms of prolongation of PFS. Indeed, in patients with classical mutations, a PFS exceeding 13 months seems superior to the 9-10 months PFS seen in trials with reversible TKIs. ${ }^{3-8,27}$ This may be due to the delay in the appearance of acquired resistance to EGFR blockade that would be a great scientific goal if confirmed in the ongoing trial. Nonetheless, erlotinib, gefitinib, and afatinib should be currently considered as alternative and equivalent front-line treatment of EGFR-mutated NSCLC in the clinical setting and one should prefer one drug rather than another on the basis of the toxicity profile, cost, and patient choice.

\section{Trials in NSCLC with acquired resistance to reversible EGFR TKIs}

Two trials addressed the question as to whether afatinib is active in patients resistant to erlotinib or gefitinib. LUXLung 1 was a Phase IIb/III study including 585 patients with advanced lung adenocarcinoma who progressed after one or two lines of chemotherapy and 12 or more weeks of either erlotinib or gefitinib treatment. ${ }^{30}$ Notably, EGFR mutational status was not an inclusion criterion. Patients were randomly allocated $(2: 1)$ to receive afatinib $50 \mathrm{mg}$ daily plus best supportive care (BSC) or placebo plus BSC. The experimental drug significantly improved PFS compared to placebo (3.3 vs 1.1 months, HR: 0.38 , 95\% CI: $0.31-0.48$; $P<0.0001) .{ }^{30}$ Moreover, responses were more frequent in the afatinib group than in the placebo group ( $7 \%$ vs $<1 \%$, respectively). ${ }^{30}$ However, the primary end point of the study was not met; median OS was 10.8 months for afatinib and 12.0 months for placebo (HR: $1.08,95 \% \mathrm{CI}$ : 0.86-1.35; $P=0.74) .{ }^{30}$ Several confounding elements can be identified in this study. First of all, it was designed on the assumption of an OS of 4.7 months in the control arm. However, this group of patients showed an extraordinary median survival of 12 months, probably due to the enrichment in tumors with less aggressive biology. Moreover, a greater proportion of patients in the placebo group received further treatment compared to the experimental arm (79\% vs $68 \%$, respectively). ${ }^{30}$ Eventually, the most impacting element in this trial is the lack of biological selection of patients. No information about $E G F R$ mutational status was required to enroll in the study and the molecular mechanism underlying the clinical resistance was unknown. Therefore, although subjects more likely to harbor EGFR mutations were clinically selected, the population was a potpourri of patients with wild-type $E G F R$ (commonly insensitive to EGFR inhibition), mutated $E G F R$, and among the last group, patients harboring several molecular mechanisms of resistance. Indeed, when a posthoc analysis was performed on patients with known EGFR mutational status who met Jackman's criteria for acquired resistance, ${ }^{31}$ translating into an enrichment of patients with real acquired resistance to EGFR TKIs, the PFS difference was more in favor of afatinib (4.5 vs 1.0 months). In addition, afatinib should be active in patients with the T790M mutation and in those with HER2 amplification, representing $60 \%-70 \%$ of the resistant population. The scientific rationale of giving afatinib to patients with other acquired molecular aberrations after resistance has occurred is not very strong. Indeed, a recent preclinical study shows how in the presence of acquired MET amplification and HGF overexpression, afatinib monotherapy is unable to inhibit the growth of cells that were originally sensitive to first-generation EGFR TKIs. ${ }^{32}$ Eight patients enrolled in the study were known to harbor a T790M mutation on archival tissue collected at diagnosis, but authors provided no information on the outcome of these patients. ${ }^{30}$ It is likely that better selection on the basis of EGFR mutational status and acquired resistance mechanism would have translated into positive results also for the principal end point (OS).

LUX-Lung 4 is an exploratory Phase II trial in Japanese patients with advanced lung adenocarcinoma who failed one to two lines of chemotherapy and progressed on erlotinib and/or gefitinib. ${ }^{33}$ In this study, EGFR status was available for a substantial proportion of patients ( 56 out of 62 patients, 90.3\%). Surprisingly, in EGFR wild type patients, RR was higher than in EGFR-mutation-positive patients (27\% vs $4.5 \%$, respectively). ${ }^{33}$ In particular, two patients were proved to harbor a concomitant T790M mutation at progression and showed stable disease for 9 months and 1 month, respectively. ${ }^{33}$ Therefore, no conclusion can be drawn for the small number of patients and the huge difference of outcome. Overall results of LUX-Lung 4 are similar to LUX-Lung 1 (RR: 8.2\%, PFS: 4.4 months, OS: 19.0 months) and in this case, the same criticisms are valid.

Considering the above mentioned potential biases of the two studies, no conclusion can be currently drawn based on LUX-Lung 1 and 4, and further studies using validated biomarkers are urgently warranted to clarify if afatinib is active in EGFR TKI-resistant patients. Encouraging data for 
the treatment of this category of NSCLC patients have come from a Phase Ib/II trial in which afatinib was combined with cetuximab. ${ }^{34}$ The trial enrolled patients with NSCLC who had acquired resistance to erlotinib or gefitinib. The rationale of the combination lies in some preclinical work in which mice with $E G F R$-mutated lung cancer and acquired resistance to EGFR TKIs showed tumor regression under treatment with afatinib and cetuximab. ${ }^{35}$ In the clinical trial, results from the first 96 evaluable patients showed impressive objective response rate (ORR) and disease control rate (DCR; 30\% and $75 \%$, respectively). ${ }^{34}$ Response to treatment was observed both in patients bearing a T790M mutation or other mechanisms of resistance.

\section{Trials in EGFR wild type NSCLC}

The LUX-Lung program does not consider studies in EGFR wild type patients. Only the LUX-Lung 8 study is enrolling patients with squamous histology that per definition should harbor no mutation in EGFR. Patients are randomized to receive either afatinib or erlotinib as second-line treatment after platinum-based chemotherapy. The trial is still ongoing and no preliminary result has yet been released. The only available data in wild type patients come from other studies sub-analysis. For example, in 45 patients enrolled in LUX-Lung 1 with no EGFR mutation, afatinib did not show a great activity profile. ${ }^{30}$ No difference in PFS was reported for the afatinib group compared to the placebo one (2.8 vs 1.8 months, HR: $0.61,95 \%$ CI: $0.27-1.35 ; P=0.22) .{ }^{30}$ Nonetheless, these results were obtained on a small number of patients and the study was not designed to address the specific question of studying afatinib in EGFR wild type patients. The results of LUX-Lung 8 will provide more robust data in the squamous histology. However, further studies are needed in EGFR wild type patients with other histology subtypes.

\section{Safety and tolerability}

\section{Toxicity profile}

Afatinib is a potent irreversible inhibitor of the tyrosine kinase activity of all the ErbB family receptors. ${ }^{19}$ EGFR and other receptors of its super-family are necessary for maintaining mucosal and skin integrity and promoting their repair. Consequently, the most common adverse effects are gastrointestinal and cutaneous, as for erlotinib and gefitinib. ${ }^{3-8}$ Moreover, given its pharmacodynamic properties, afatinib may exhibit more serious toxicity compared to reversible EGFR TKIs. Data from the three major Phase III trials (LUX-Lung 1, 3, and 6) will be used to evaluate these two aspects of afatinib tolerability (Table 3). ${ }^{24,25,30}$

The most frequent adverse effects in afatinib trials were diarrhea and cutaneous rash, occurring in $87 \%-96 \%$ and $78 \%-90 \%$ of patients, respectively. ${ }^{24,25,30}$ Although almost all patients experienced at least one of these side effects during the treatment, only $6 \%-17 \%$ of them presented grade $\geq 3$ diarrhea and $14 \%-16 \%$ grade $\geq 3$ skin rash. ${ }^{24,25,30}$ Other gastrointestinal effects were stomatitis, nausea, vomiting, and decreased appetite. Paronychia also appeared frequently. All these side effects were also reported in trials evaluating reversible EGFR TKIs, ${ }^{3-8}$ but the general perception is that on afatinib, they occur at somewhat higher rates and are generally worse than those observed with erlotinib and gefitinib. For example, the percentage of patients experiencing any grade of diarrhea under erlotinib treatment was $57 \%$ in the EURTAC (EURopean TArceva vs Chemotherapy) trial and $25 \%$ in the OPTIMAL trial. ${ }^{7,8}$ In the same studies, grade $\geq 3$ diarrhea was observed in only $5 \%$ and $1 \%$ of patients, respectively. ${ }^{7,8}$ Also, paronychia and stomatitis were more frequent and serious in afatinib studies than in trials with reversible TKIs. No clear difference was observed in skin rash incidence on erlotinib or afatinib. In LUX-Lung 2, patients

Table 3 Toxicity profile in the major Phase III clinical trials of afatinib, erlotinib, and gefitinib

\begin{tabular}{|c|c|c|c|c|c|c|c|c|c|c|}
\hline & \multicolumn{2}{|c|}{$\begin{array}{l}\text { LUX-Lung } \mathrm{I}^{30} \\
\text { (Afatinib } 50 \mathrm{mg} \text { ) }\end{array}$} & \multicolumn{2}{|c|}{$\begin{array}{l}\text { LUX-Lung } 3^{24} \\
\text { (Afatinib } 40 \mathrm{mg} \text { ) }\end{array}$} & \multicolumn{2}{|c|}{$\begin{array}{l}\text { LUX-Lung } 6^{25} \\
\text { (Afatinib } 40 \mathrm{mg} \text { ) }\end{array}$} & \multicolumn{2}{|c|}{$\begin{array}{l}\text { EURTAC }^{8} \\
\text { (Erlotinib I50 mg) }\end{array}$} & \multicolumn{2}{|c|}{$\begin{array}{l}\text { IPASS }^{3} \\
\text { (Gefitinib } 250 \mathrm{mg} \text { ) }\end{array}$} \\
\hline & All & $\geq$ Grade 3 & All & $\geq$ Grade 3 & All & $\geq$ Grade 3 & All & $\geq$ Grade 3 & All & $\geq$ Grade 3 \\
\hline Diarrhea & $87 \%$ & $17 \%$ & $96 \%$ & $15 \%$ & $90 \%$ & $6 \%$ & $57 \%$ & $5 \%$ & $47 \%$ & $4 \%$ \\
\hline Rash/acne & $78 \%$ & $14 \%$ & $90 \%$ & $16 \%$ & $81 \%$ & $15 \%$ & $80 \%$ & $13 \%$ & $66 \%$ & $3 \%$ \\
\hline Stomatitis/mucositis & $61 \%$ & $3 \%$ & $73 \%$ & $9 \%$ & $52 \%$ & $5 \%$ & NR & NR & $17 \%$ & 0 \\
\hline Paronychia & $39 \%$ & $5 \%$ & $57 \%$ & II\% & $33 \%$ & $0 \%$ & NR & NR & $14 \%$ & 0 \\
\hline Decreased appetite & $31 \%$ & $4 \%$ & $29 \%$ & $4 \%$ & $16 \%$ & $3 \%$ & $31 \%$ & 0 & $22 \%$ & $2 \%$ \\
\hline Nausea & $23 \%$ & $2 \%$ & $25 \%$ & $1 \%$ & $12 \%$ & $0 \%$ & NR & NR & $17 \%$ & 0 \\
\hline Fatigue & $29 \%$ & $6 \%$ & $27 \%$ & $3 \%$ & $17 \%$ & $2 \%$ & $57 \%$ & $6 \%$ & $17 \%$ & 0 \\
\hline Vomiting & $20 \%$ & $2 \%$ & $23 \%$ & $4 \%$ & $13 \%$ & $1 \%$ & NR & NR & $13 \%$ & 0 \\
\hline
\end{tabular}

Abbreviations: NR, not reported; EURTAC, European Erlotinib versus chemotherapy trial; IPASS, Iressa Pan Asia Study. 
were treated with either afatinib $50 \mathrm{mg}$ or $40 \mathrm{mg}$. The toxicity profile was slightly better for afatinib at a lower dosage with no difference in activity. ${ }^{23}$ In particular, lowering the dose resulted in a reduction of grade $\geq 3$ diarrhea (50 $\mathrm{mg}$ vs 40 mg: $22 \%$ vs $7 \%$ ) and skin rash (50 mg vs $40 \mathrm{mg}: 28 \%$ vs $7 \%$ ) with no difference in the overall incidence of side effects. ${ }^{23}$ Despite the high percentage of adverse events reported, only $8 \%$ of patients receiving afatinib discontinued the treatment in LUX-Lung 1 and 3. ${ }^{24,30}$ In LUX-Lung 6, this percentage was even lower $(5.9 \%) .{ }^{25}$ In conclusion, it seems that a higher incidence of side effects is the price to pay for stronger activity. However, the low percentage of patients discontinuing the drug suggests that the reported side effects may be easily managed and solved.

\section{Management of adverse events}

As discovered in the past few years with reversible EGFR TKIs and even more so for irreversible TKIs, treatment of adverse events should be proactive and should start as soon as possible after the onset of symptoms. The first step to avoid serious side effects is education of patients. In particular, a patient should be well-informed on the frequency and potential intensity of specific events, the purpose of the treatment of such side effects, and the implications of delayed treatment.

Skin rash is the most common adverse event with EGFR inhibitors and is usually localized to the face and trunk. Other cutaneous side effects may include erythema, pruritus, xerosis, and paronychia. ${ }^{3-8,24,25,30}$ Skin effects may improve or completely resolve with continued use of afatinib, although sometimes dose reduction or drug discontinuation are necessary. Patients should be informed on prevention measures to reduce the risk of skin effects. Firstly, intense exposure to sunlight should be avoided and sunscreen should be used on exposed areas (face, neck, arms, legs) regardless of season. Moreover, patients should wear protective clothing and avoid using harsh detergents. When a skin rash is already onset, treatment should be given. In the case of grade 1-2 rash, afatinib should be continued at the current dose and topical treatment with steroids and antibiotics should be prescribed ${ }^{36}$ In the case of grade 2 rash, oral antibiotics for 6 weeks may be considered instead of topical therapy. ${ }^{36}$ In the case of grade 3 skin rash, afatinib should be interrupted and the patient should be referred to a dermatologist or given oral antibiotics for 6 weeks. ${ }^{36}$ Afatinib should be restarted at a reduced dose $(10 \mathrm{mg})$ if the patient recovers to grade $1 .{ }^{36}$
Diarrhea usually appears within the first week from the start of afatinib treatment. Early recognition and management of diarrhea is essential to prevent dose reductions or discontinuation. Patients should be given anti-diarrheal agents (loperamide) when afatinib is started and be advised to take this medication immediately with the onset of diarrhea. ${ }^{37}$ Moreover, patients should be advised to drink an adequate quantity of fluids (8-10 glasses of clear fluids) to balance the loss through diarrhea and should be given strict dietary recommendations. In particular, in the case of grade 1-2 diarrhea, loperamide should be started immediately (4 mg followed by $2 \mathrm{mg}$ after each loose stool, with up to $16 \mathrm{mg}$ total daily) until bowel movements cease for 12 hours. ${ }^{37}$ In the case of grade 2 diarrhea, electrolytes should be assessed to avoid potential imbalances. When grade $\geq 3$ diarrhea is present, hospitalization is recommended for aggressive intravenous fluid replacement and stool cultures. ${ }^{37}$ Prophylactic antibiotics should be considered if the patient is neutropenic. ${ }^{37}$

\section{Patients' perspectives}

Despite the high frequency and strong intensity of adverse events, afatinib treatment improves patients' reported quality of life. In LUX-Lung 1, afatinib plus BSC was compared to placebo plus BSC. Symptoms and health-related QoL were evaluated using the lung cancer-specific European Organization for Research and Treatment of Cancer (EORTC) Quality of life questionnaire C30 (QLQ-C30) and Lung Cancer 13 (LC13) and EuroQol (EQ-5D) questionnaires at baseline and every 21 days. ${ }^{38}$ A significantly higher proportion of patients in the afatinib arm showed improvement in cough $(P<0.0001)$, dyspnea $(P=0.006)$, and pain $(P<0.0001)$ compared to placebo. ${ }^{38}$ Moreover, afatinib significantly delayed the time to deterioration for cough $(P<0.001)$, but showed only a trend in delaying dyspnea $(P=0.170)$, and pain $(P=0.287) .{ }^{38}$ In the experimental arm, patients reported worse diarrhea, sore mouth, and appetite due to the toxicity profile of the compound. However, afatinib significantly improved QoL in the EQ-5D questionnaire assessment and global health status/QoL, physical functioning, and fatigue in the assessment through EORTC questionnaires. ${ }^{38}$ In conclusion, afatinib improved symptoms and QoL of patients compared to placebo.

The same beneficial effect of afatinib on symptoms and QoL was reported when the drug was compared to chemotherapy in LUX-Lung 3 and $6 .^{39,40}$ In both trials, the patients' reported outcomes were assessed using the EORTC QLQ-C30 and LC13 at baseline and every 21 days. 
In LUX-Lung 3, a higher proportion of patients in the afatinib arm experienced improvement in dyspnea compared to chemotherapy $(P=0.010) .{ }^{39}$ In addition, afatinib significantly delayed the time to deterioration for cough $(P=0.007)$ and dyspnea $(P=0.015)$, but not pain $(P=0.19) .{ }^{39}$ Afatinib significantly improved global health status/QoL $(P=0.015)$ and physical $(P<0.001)$, role $(P=0.004)$, and cognitive $(P=0.007)$ functioning compared to chemotherapy. Fatigue and nausea were worse with chemotherapy, whereas diarrhea $(P<0.01)$, dysphagia $(P<0.01)$, and sore mouth $(P<0.01)$ were worse with afatinib. ${ }^{39}$ Similarly, in LUX-Lung 6, more patients on afatinib had improvement of cough $(P=0.0003)$, dyspnea $(P<0.0001)$, and pain $(P=0.003)$ compared to chemotherapy. ${ }^{40}$ Moreover, afatinib delayed the time to deterioration for cough $(P=0.0001)$, dyspnea $(P<0.0001)$, and pain $(P=0.03) .{ }^{40}$ A significantly higher percentage of patients experienced worsening diarrhea, sore mouth, and dysphagia with afatinib, while fatigue, nausea, and vomiting were worse with chemotherapy. ${ }^{40}$ However, afatinib improved QoL $(P<0.0001)$, physical $(P<0.0001)$, role $(P=0.01)$, and social $(P<0.001)$ functioning compared to chemotherapy. ${ }^{40}$

In general, in these studies, patients reported better outcomes if treated with afatinib rather than placebo or platinumbased chemotherapy. In addition, afatinib gave significantly longer control of lung cancer-related symptoms compared to placebo or chemotherapy. This effect was expected, as it is widely known from previous studies that EGFR inhibition can drastically and quickly improve QoL and functioning of patients with EGFR-addicted lung tumors. It will be interesting to see how afatinib performs in terms of symptoms and QoL improvement when compared head-to-head to reversible EGFR TKIs in the already ongoing clinical trials.

\section{Conclusion}

Several trials evaluating afatinib in the clinical setting have been published, and preliminary results are available for others. Therefore, initial recommendations for the use of this agent can be drawn. Afatinib is a very encouraging drug for EGFR-mutation-positive advanced lung cancer. Indeed, the studies LUX-Lung 3 and 6 showed that afatinib is superior to chemotherapy in terms of RR, PFS, and QoL. In these trials, PFS seems to be prolonged compared to reversible TKIs. However, only the results of the LUX-Lung 7 trial will establish if afatinib is better or equal to gefitinib. A headto-head trial comparing afatinib to erlotinib is still missing. Considering that it is at a minimum equivalent to reversible TKIs, afatinib should be currently considered an alternative drug to use for front-line treatment of $E G F R$-mutated patients.
In particular, afatinib should be used in patients with classical mutations in the EGFR gene (exon 19 deletions and exon 21 point mutations) and could be considered in the presence of G719X and L861Q mutations. Patients with exon 20 insertions and de novo T790M should best be treated with chemotherapy or included in clinical trials with novel agents. Afatinib also showed some activity in patients with EGFR mutations who are resistant to both chemotherapy and reversible TKIs, but the study that sought to address this question has several biases that reduce the strength of its results. Nevertheless, for this specific population of NSCLC patients, there is no treatment of proven efficacy and the 3-4 months prolongation of PFS obtained with afatinib should not be disregarded. In EGFR wild type advanced NSCLC, data are still too preliminary for any kind of statement.

Taken together, afatinib may be considered as a useful potential therapeutic agent that deserves to be better studied both in EGFR-mutated patients with acquired resistance to TKIs and in the EGFR wild type population. To date, no targeted drug given as a single agent has been able to cure any cancer patients. Therefore, in our opinion, studies evaluating combinations of afatinib with other targeted agents are warranted. The history of afatinib demonstrates how knowledge of the molecular aspects of tumors may help researchers to rationally develop new treatment strategies in the preclinical setting and improve treatment outcomes in clinic. However, future studies should require the mandatory collection of tissue at study entry and at progression to better define groups of patients who would benefit more from afatinib and the mechanisms of acquired resistance to this interesting drug.

\section{Disclosure}

The authors report no conflicts of interest in this work.

\section{References}

1. Rowinski EK. The erbB family: targets for therapeutic development against cancer and therapeutic strategies using monoclonal antibodies and tyrosine kinase inhibitors. Annu Rev Med. 2004;55:433-457.

2. Kumar A, Petri ET, Halmos B, et al. Structure and relevance of the epidermal growth factor receptor in human cancer. J Clin Oncol. 2008;26:1742-1751.

3. MokTS, Wu YL, Thongprasert S, et al. Gefitinib or carboplatin-paclitaxel in pulmonary adenocarcinoma. N Engl J Med. 2009;361:947-957.

4. Lee JS, Park K, Kim S-W, et al. A randomized phase III study of gefitinib (IRESSA) versus standard chemotherapy (gemcitabine plus cisplatin) as a first-line treatment for never-smokers with advanced or metastatic adenocarcinoma of the lung. JThor Oncol. 2009;4(Suppl 19): abst PRS 4.

5. Mitsudomi T, Morita S, Yatabe Y, et al. Gefitinib versus cisplatin plus docetaxel in patients with non-small-cell lung cancer harbouring mutations of the epidermal growth factor receptor (WJTOG3405): an open label, randomised phase 3 trial. Lancet Oncol. 2010;11:121-128. 
6. Maemondo M, Inoue A, Kobayashi K, et al. Gefitinib or chemotherapy for non-small-cell lung cancer with mutated EGFR. $N$ Engl J Med. 2010;362:2380-2388.

7. Zhou CC, Wu YL, Chen G, et al. Erlotinib versus chemotherapy as first-line treatment for patients with advanced EGFR mutation-positive non-small-cell lung cancer (OPTIMAL, CTONG-0802): a multicentre, open-label, randomised, phase 3 study. Lancet Oncol. 2011;12: 735-742.

8. Rosell R, Carcereny E, Gervais R, et al. Erlotinib versus standard chemotherapy as first-line treatment for European patients with advanced EGFR mutations-positive non-small cell lung cancer (EURTAC) a multicenter, open-label, randomized phase 3 trial. Results of the European Erlotinib Versus Chemotherapy (EURTAC) phase III randomized trial. Lancet Oncol. 2012;13(3):239-246.

9. Kobayashi S, Boggon TJ, Dayaram T, et al. EGFR mutation and resistance of non-small-cell lung cancer to gefitinib. $N$ Engl J Med. 2005;352:786-792.

10. Balak MN, Gong Y, Riely GJ, et al. Novel D761Y and common secondary T790M mutations in epidermal growth factor receptor-mutant lung adenocarcinoma with acquired resistance to kinase inhibitors. Clin Cancer Res. 2006;12:6494-6501.

11. Engelman JA, Zajnullahu K, Mitsudomi T, et al. MET amplification leads to gefitinib resistance in lung cancer by activating ERBB3 signaling. Science. 2007;316:1039-1043.

12. Yu HA, Arcila ME, Rekhtman N, et al. Analysis of tumor specimens at the time of acquired resistance to EGFR-TKI therapy in 155 patients with EGFR-mutant lung cancers. Clin Cancer Res. 2013;19(8): 2240-2247.

13. Sequist LV, Waltman BA, Dias-Santagata D, et al. Genotypic and histological evolution of lung cancers acquiring resistance to EGFR inhibitors. Sci Transl Med. 2011;3:75ra26.

14. Kosaka T, Yatabe Y, Endoh H, et al. Analysis of epidermal growth factor receptor gene mutation in patients with non-small cell lung cancer and acquired resistance to gefitinib. Clin Cancer Res. 2006;12:5764-5769.

15. Pao W, Miller VA, Politi KA, et al. Acquired resistance of lung adenocarcinomas to gefitinib or erlotinib is associated with a second mutation in the EGFR kinase domain. PLoS Med. 2005;225;2(3):e73.

16. Godin-Heymann N, Bryant I, Rivera MN, et al. Oncogenic activity of epidermal growth factor receptor kinase mutant alleles is enhanced by the T790M drug resistance mutation. Cancer Res. 2006;67:7319-7326.

17. Inukai $\mathrm{M}$, Toyooka $\mathrm{S}$, Ito $\mathrm{S}$, et al. Presence of epidermal growth factor receptor gene $\mathrm{T} 790 \mathrm{M}$ mutation as a minor clone in non-small cell lung cancer. Cancer Res. 2008;266:7854-7858.

18. Li D, Ambrogio L, Shimamura T, et al. BIBW2992, an irreversible EGFR/HER2 inhibitor highly effective in preclinical lung cancer models. Oncogene. 2008;27(34):4702-4711.

19. Solca F, Dahl G, Zoephel A, et al. Target binding properties and cellular activity of afatinib (BIBW 2992), an irreversible ErbB family blocker. J Pharmacol Exp Ther. 2012;343(2):342-350.

20. Stopfer P, MArzin K, Narjes H, et al. Afatinib pharmacokinetics and metabolism after oral administration to healthy male volunteers. Cancer Chemother Pharmacol. 2012;69(4):1051-1061.

21. Yap TA, Vidal L, Adam J, et al. Phase I trial of the irreversible EGFR and HER2 kinase inhibitor BIBW 2992 in patients with advanced solid tumors. J Clin Oncol. 2010;28(25):3965-3972.

22. Wind S, Schmid M, Erhardt J, Goeldner RG, Stopfer P. Pharmacokinetics of afatinib, a selective irreversible ErbB family blocker, in patients with advanced solid tumours. Clin Pharmacokinet. 2013;52(12):1101-1109.

23. Yang JC, Shih JY, Su WC, et al. Afatinib for patients with lung adenocarcinoma and epidermal growth factor receptor mutations (LUX-Lung 2): a phase 2 trial. Lancet Oncol. 2012;13(5):539-548.

24. Sequist LV, Yang JC, Yamamoto N, et al. Phase III study of afatinib or cisplatin plus pemetrexed in patients with metastatic lung adenocarcinoma with EGFR mutations. J Clin Oncol. 2013;31(27):3327-3334.
25. Wu YL, Zhou C, Hu CP, et al. LUX-Lung 6: a randomized, open-label, phase III study of afatinib (A) versus gemcitabine/cisplatin (GC) as first-line treatment for Asian patients (pts) with EGFR mutation-positive (EGFR M+) advanced adenocarcinoma of the lung. J Clin Oncol. 2013;31(Suppl): abst 8016.

26. FDA. Summary of Safety and Effectiveness Data (SSED). http:// www.accessdata.fda.gov/cdrh_docs/pdf12/P120022b.pdf. Accessed February 11, 2014.

27. Yang JC-H, Sequist LV, Geater SL, et al. Activity of afatinib in uncommon epidermal growth factor receptor (EGFR) mutations: findings from three trials of afatinib in EGFR mutation-positive lung cancer. Oral presentation at: World Congress on Lung Cancer; October 27-31; 2013; Sydney, Australia.

28. Wu JY, Yu CJ, Chang YC, et al. Effectiveness of tyrosine kinase inhibitors on "uncommon" epidermal growth factor receptor mutations of unknown clinical significance in non-small cell lung cancer. Clin Cancer Res. 2011;17(11):3812-3821.

29. Boehringer Ingelheim. LUX-Lung 7: a phase IIb trial of afatinib (BIBW2992) versus gefitinib for the treatment of 1st line EGFR mutation positive adenocarcinoma of the lung. Available from: http://clinicaltrials.gov/ct2/show/NCT01466660. NLM identifier: NCT01466660. Accessed October 21, 2013.

30. Miller VA, Hirsh V, Cadranel J, et al. Afatinib versus placebo for patients with advanced, metastatic non-small-cell lung cancer after failure of erlotinib, gefitinib, or both, and one or two lines of chemotherapy (LUX-Lung 1): a phase 2b/3 randomised trial. Lancet Oncol. 2012;13(5):528-538.

31. Jackman D, Pao W, Riely GJ, et al. Clinical definition of acquired resistance to epidermal growth factor receptor tyrosine kinase inhibitors in non-small-cell lung cancer. J Clin Oncol. 2010;28(2): $357-360$.

32. Nanjo S, Yamada T, Nishihara H, et al. Ability of the Met kinase inhibitor crizotinib and new generation EGFR inhibitors to overcome resistance to EGFR inhibitors. PLoS One. 2013;8(12):e84700.

33. Katakami N, Atagi S, Goto K, et al. LUX-Lung 4: a phase II trial of afatinib in patients with advanced non-small-cell lung cancer who progressed during prior treatment with erlotinib, gefitinib or both. J Clin Oncol. 2013;31(27):3335-3341.

34. Janjigian YY, Groen HJ, Horn L, et al. Activity and tolerability of afatinib (BIBW 2992) and cetuximab in NSCLC patients with acquired resistance to erlotinib or gefitinib. J Clin Oncol. 2011;29(Suppl): abst 7525 .

35. Regales L, Gong Y, Shen R, et al. Dual targeting of EGFR can overcome a major drug resistance mutation in mouse models of EGFR mutant lung cancer. J Clin Invest. 2009;119(10):3000-3010.

36. Lacouture ME, Schadendorf D, Chu CY, et al. Dermatologic adverse events associated with afatinib: an oral ErbB family blocker. Expert Rev Anticancer Ther. 2013;13(6):721-728.

37. Yang JC, Reguart N, Barinoff J, et al. Diarrhea associated with afatinib: an oral ErbB family blocker. Expert Rev Anticancer Ther. 2013;13(6): 729-736.

38. Hirsh V, Cadranel J, Cong XJ, et al. Symptom and quality of life benefit of afatinib in advanced non-small-cell lung cancer patients previously treated with erlotinib or gefitinib: results of a randomized phase IIb/III trial (LUX-Lung 1). J Thorac Oncol. 2013;8:229-237.

39. Yang JC, Hirsh V, Schuler M, et al. Symptom control and quality of life in LUX-Lung 3: a phase III study of afatinib or cisplatin/pemetrexed in patients with advanced lung adenocarcinoma with EGFR mutations. J Clin Oncol. 2013;31(27):3342-3350.

40. Geater SL, Zhou C, Hu C, et al. LUX-Lung 6: Patient-reported outcomes (PROs) from a randomized open-label, phase III study in first-line advanced NSCLC patients (pts) harboring epidermal growth factor receptor (EGFR) mutations. Abstract at: World Congress on Lung Cancer; October 27-31; 2013; Sydney, Australia; abst 8061. 


\section{Publish your work in this journal}

Biologics: Targets \& Therapy is an international, peer-reviewed journal focusing on the patho-physiological rationale for and clinical application of Biologic agents in the management of autoimmune diseases, cancers or other pathologies where a molecular target can be identified. This journal is indexed on PubMed Central, CAS, EMBase, Scopus

and the Elsevier Bibliographic databases. The manuscript management system is completely online and includes a very quick and fair peerreview system, which is all easy to use. Visit http://www.dovepress. com/testimonials.php to read real quotes from published authors. 